\title{
CONTRIBUTION TO THE STUDY OF THE AUTONOMIC NERVOUS SYSTEM IN CHILDREN
}

BY

\author{
A. J. CHURA, M.D.
}

\author{
Physician to the University Children's Clinic, Bratislava.
}

The observations of E. F. Müller. (1) on the decrease in number of the white blood-cells in the peripheral blood consequent upon certain forms of skin irritation have given rise to a heated debate. Some authors are of opinion that this reaction is due to a biological action of the skin; others compare Müller's results with Widal's " hæmoclastic crisis"; others, again, think the change in the number of leucocytes is produced differently in healthy and in sick persons, obeying no rule.

There are many different ways in which the change in the number of leucocytes can be brought about. E. F. Müller gave intradermal injections of inert and of physiological solutions, and even of distilled water. Others injected hypertonic and hypotonic solutions, and solutions of the salts of potassium, calcium, magnesium, etc., and observed the change in the number of leucocytes following such injections. Other ways of obtaining the reaction are :-injection of cooled solutions, painful in themselves; cauterization of the skin by the application of concentrated acids or alkalies ; causing pain by pricking the finger-tips, etc. It is also possible to produce the same reaction by freezing the skin with ethyl chloride, as in the production of local anæsthesia. These irritants, for the most part act on the sensory nerve endings, and by the same means cause different reactions.

The first to make these discoveries was E. F. Müller. He noted that a more or less rapid decrease in the number of leucocytes followed intradermal injections even of inert solutions, the decrease in the peripheral blood being very marked in certain cases. By further experiments he found that, simultaneously with the peripheral leucopenia, a dilatation of vessels and a leucocytosis occurred in the liver and mesentery; whereas a peripheral leucocytosis was accompanied by vaso-constriction and leucopenia in the liver and mesentery. If such be the case, as vaso-dilatation results from parasympathetic influence, and vaso-constriction from sympathetic influence, I think that the impulse must run through a reflex arc, beginning in the skin and ending in the terminations of the vegetative nerves. In the whole reaction the following are called into play :-

1. The skin, with the nerve-endings in it.

2. The centripetal arm of the reflex arc (the sensory nerve or vegetative nerves of skin and vessels).

3. The connection between centripetal and centrifugal arm.

4. The centrifugal arm (the autonomic nervous system of the "cavum abdominis" or of the vegetative organs in general).

5. The terminations of the vegetative nerves in the blood-vessel and in the vegetative organs in general. 
6. The physico-chemical medium.

7. The white blood-cells, and their number, under certain conditions. Also, certain vegetative centres.

A somewhat similar reflex is concerned in the secretion of gastric juice and of saliva in response to ocular, olfactory or acoustic stimulation.

After due consideration of these statements, I examined a number of children according to these principles. The method was as follows:-The children were examined in the morning before breakfast, or in the afternoon some hours after dinner. Before and during the examination the child was kept in bed and care was taken not to excite nor disturb it in any way. Blood was taken in the usual way from the finger-tip. After the number of leucocytes had been determined, the skin of the upper arm or thigh was sprayed with ethyl chloride until it was frozen over an area of 2 to $3 \mathrm{~cm}$., and the number of leucocytes was determined in the blood taken every ten minutes from the moment of freezing. The blood was taken six or seven times from the same incision, and with the same instrument. This method was chosen because the child was least disturbed by it, and it usually had no psychical effect ; further, the degree of irritation oan be accurately adjusted, and is automatically adapted to the temperature of the body and the surroundings.

For my first experiments, I chose cases of measles and typhoid fever ; consequently, leucopenia was present. Ten cases of measles were examined 22 times, and 17 cases of typhoid 28 times.

In the developing stages of measles, and while the rash was first appearing, a decrease of $18-50 \%$ in the number of leucocytes was noticeable as a rule; an average of $29.5 \%$. From the moment the rash began to fade, an increase in the number of leucocytes was usually apparent on irritation of the skin ; here, again, from 18 to $48 \%$, an average of $32.5 \%$.

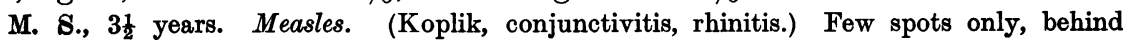
he ears and on the chest.

\begin{tabular}{|c|c|c|c|c|}
\hline Before freezing & .. & $\cdots$ & $\cdots$ & 6450 \\
\hline After freezing 10 & minutes & .. & .. & 3600 \\
\hline 20 & " & .. & . & 4850 \\
\hline 30 & ", & $\ldots$ & $\ldots$ & 4800 \\
\hline 40 & " & .. & . & 3200 \\
\hline 50 & ", & .. & .. & 6050 \\
\hline 60 & $"$ & .. & . & 6200 \\
\hline
\end{tabular}

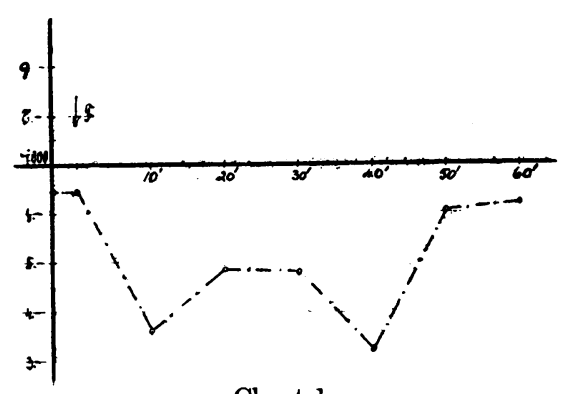

Chart 1, 
Same patient three days later; the whole body covered with a thick rash, confluent in some places, in the fading stage.

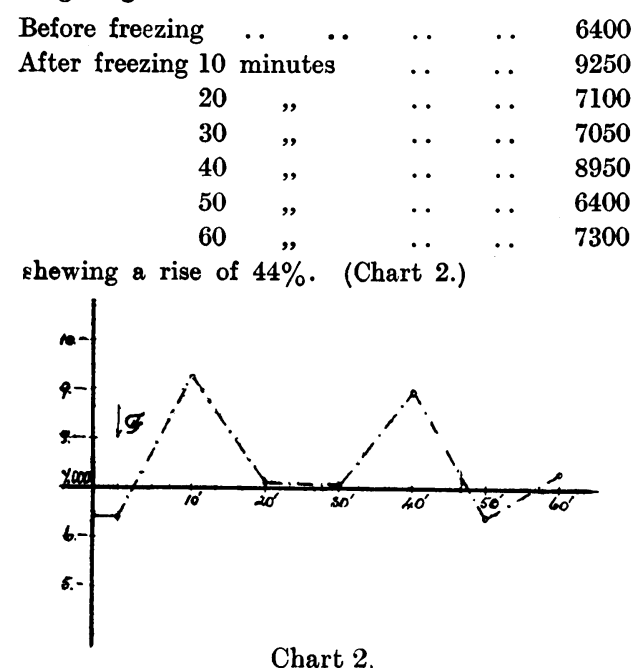

Chart 2

Since in all the other cases the same course was observed, I refrain from giving further examples.

In cases of typhoid fever, an average decrease in the number of leucocytes of $32 \%$ was, as a rule, noticeable during the whole course of the disease.

A. B., 11 years. Typhoid Fever.

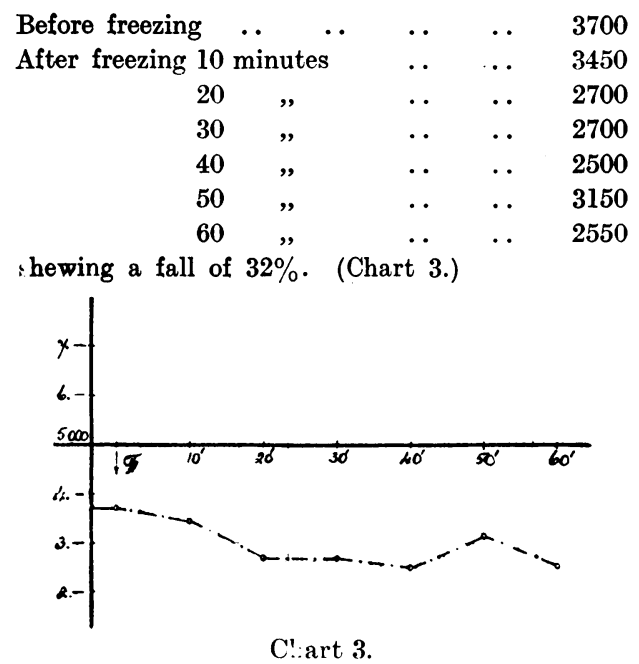

During these experiments I proved that the tests could only be carried out, in cases of typhoid, when the temperature of the patients was not high. Possibly irritation of the sympathetic centres was caused by the pyrexia or by acidosis. 
I followed up these two leucopenic diseases and proved that such simple irritation produced a fluctuation in the number of leucocytes which varied with the disease, and with the stage of the disease, but not with the individual.

It is not possible to ascribe this fluctuation, on such slight irritation, to an increased formation or destruction of the white blood-corpuscles; for it there must be some other explanation. As already mentioned, E. F. Müller found, simultaneously with peripheral leucopenia, a dilatation of the vessels of the liver and splanchnic region and an accumulation of leucocytes in this region: with peripheral leucocytosis, he found vaso-constriction and leucopenia in the splanchnic area. It is a well-known fact that with enlargement of the lumen the rate of flow diminishes, and that friction varies inversely as the rate of flow. Further, elements of lower specific gravity accumulate in the peripheral zone of the blood-stream and those of higher specific gravity in the centre. As the leucocytes are lighter, they accumulate in the peripheral zone, and there they increase when the vessel dilates, on account of their viscosity and the increased friction. In consequence of this, the flow of leucocytes to the heart is diminished, and thus fewer come into the general circulation. The contrary happens in consequence of contraction of the vessels in a more extensive region. This mechanical process may not be enough to account for the increase or decrease in the number of leucocytes in certain regions. This regulation can hardly be caused by purely physical means. The idea is very attractive that it might be brought about by the influence of the vegetative nerves. For, where a constriction of the vessels occurs, occasioned by the influence of the sympathetic nerves, the number of leucocytes decreases at the same time, as if the impulses emanating from the irritated sympathetic metabolites drove away the leucocytes. On the other hand, where vaso-dilatation takes place under the influence of the parasympathetic, an increase in the number of leucocytes is noticeable; thus, the irritated state of the parasympathetic system reacts on the leucocytes, causing them to accumulate in the irritated area. The reaction varies in intensity with the capacity of the blood-vessels in the region affected.

Constriction and dilatation of blood-vessels is controlled by the sympathetic and parasympathetic nerves. If this be so, the whole of the above-mentioned reaction works through these two systems. Thus we find also leucopenia in the blood-vessels of the ear and leucocytosis in those of the mesentery on irritation of the vagus (in dogs) by electrical methods, and the reverse reaction on irritation of the splanchnic nerves (W. Filiuski $\left.{ }^{2}\right)$ ). Similar results are observed following the injection of sympathetic or parasympathetic poisons. But one must be careful in criticizing the results of injection of these poisons. Every irritant has an effect not merely on one system but on both ; so have the poisons. Since the effect is on both, the degree of irritation may not be equal, nor does it necessarily take effect at the same time; the result varying with the dose and the susceptibility of the individual. The more complicated the mechanism by which the results are brought about, the more difficult it is to account for them and to draw correct deductions. The phenomena 
described are not merely a demonstration of the parasympathetic and sympathetic systems, but depend upon a conglomeration of different influences which can only be isolated with difficulty owing to lack of control over the stimulus. All this must be borne in mind in the pharmaco-dynamic examination of these systems. I always employed the freezing process, because I thought the reaction caused thereby is not dependent on the impulse nor on the irritant itself, but merely on the state of both systems and on their relation to each other.

Twelve children with an artificial alkalosis caused by $\mathrm{NaHCO}_{3}$, and seven with an artificial acidosis from $\mathrm{NH}_{4} \mathrm{Cl}$, were examined by the above method. If the results obtained from this method may be regarded as a criterion of the state of the vegetative nervous system, then in alkalosis there must be a decrease in the number of white corpuscles in the peripheral blood, and in acidosis an increase, for it is an established fact that parasympathetic influence is associated with an increase of blood-alkalinity and sympathetic influence with a decrease of it (K. Dresel( $\left.{ }^{3}\right)$ ). My expectations were not disappointed. The experiment was carried out in the following manner :-The reaction of the vegetative nervous system was ascertained in the morning before breakfast, and afterwards the patient was given three doses of $\mathrm{NaHCO}_{3}$, each dose amount. ing to $1-1.5 \mathrm{~g}$. per $10 \mathrm{Kg}$. of weight, and the following morning an hour before the experiment a further dose of $1-1.5 \mathrm{~g}$. per $10 \mathrm{Kg}$. of weight was administered. The test was also made with $\mathrm{NH}_{4} \mathrm{Cl}$. The experiment showed that acidosis or alkalosis caused in this manner can often diminish and even change the parasympathetic reaction into sympathetic, and vice versa. The reactions can also be intensified. It must be mentioned that the administration of $\mathrm{NH}_{4} \mathrm{Cl}$ may cause unpleasant symptoms such as nausea and vomiting, which may vitiate the whole experiment; it is, therefore, advisable to select for examination cases which are not subject to such symptoms. If it should happen that the reaction does not fulfil one's expectations, it is necessary to assure oneself that the condition of the organ under observation is such as to permit of alteration in the reaction. With regard to the importance of this point it is enough to mention the experiments on the hypogastric nerve of pregnant and non-pregnant cats : the gravid uterus responds to stimulation of this nerve with contraction, the non-gravid with relaxation (E. Schilf( $\left.{ }^{4}\right)$ ).

As all experiments had the same result, I mention only the two following cases:-

H. B., $5 \frac{1}{2}$ years. Faucial diphtheria.

\begin{tabular}{|c|c|c|c|c|}
\hline Before freezing & & . & . & 5650 \\
\hline After freezing 10 & nutes & .. & .. & 7050 \\
\hline 20 & , & .. & .. & 7250 \\
\hline 30 & , & .. & .. & 6600 \\
\hline 40 & , & .. & .. & 7350 \\
\hline 50 & , & .. & .. & 8050 \\
\hline 60 & ", & .. & .. & 6300 \\
\hline
\end{tabular}




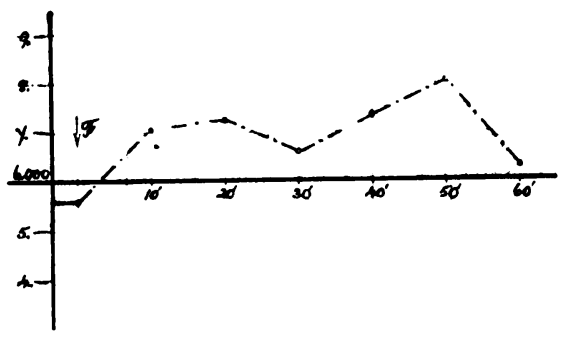

Chart 4.

The same day $7 \mathrm{~g}$. of $\mathrm{NaHCO}_{3}$ was given in three doses. The following day a dose of $3 \mathrm{~g}$. was administered, an hour before the experiment was made.

\begin{tabular}{|c|c|c|c|c|}
\hline \multirow{2}{*}{\multicolumn{2}{|c|}{$\begin{array}{l}\text { Before freezing } \ldots \\
\text { After freezing } 10 \text { minutes }\end{array}$}} & .. & $\ldots$ & 6750 \\
\hline & & .. & .. & 5600 \\
\hline 20 & , & .. & . & 6700 \\
\hline 30 & , & . & . & 5800 \\
\hline 40 & , & .. & .. & $\mathbf{5 2 5 0}$ \\
\hline 50 & , & .. & $\ldots$ & 5450 \\
\hline 60 & , & .. & $\ldots$ & 5800 \\
\hline
\end{tabular}

A decrease of $\mathbf{2 3 . 7 1 \%}$ is shown. (Chart 5.)

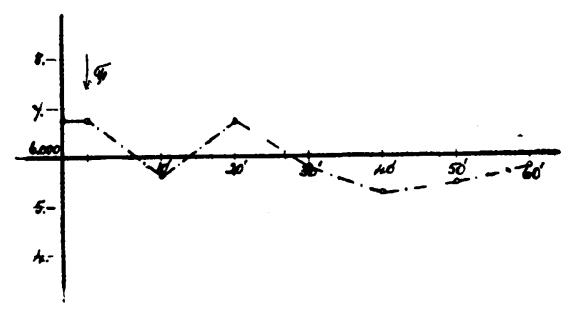

Chart 5.

H. B., 6 years. Artificial acidosis. Convalescent from diphtheria.

\begin{tabular}{|c|c|c|c|c|c|}
\hline \multirow{2}{*}{\multicolumn{3}{|c|}{$\begin{array}{l}\text { Before freezing } \ldots \\
\text { After freezing } 10 \text { minutes }\end{array}$}} & .. & . & 9650 \\
\hline & & & .. & . & 8850 \\
\hline 20 & , & & .. & $\ldots$ & 9500 \\
\hline 30 & , & .. & $\ldots$ & . & 8750 \\
\hline 40 & ", & & .. & $\ldots$ & 7700 \\
\hline 50 & ", & & .. & .. & 7950 \\
\hline 60 & , & & .. & .. & 8950 \\
\hline
\end{tabular}

shewing a fall of $20 \cdot 2 \%$. (Chart 6.)

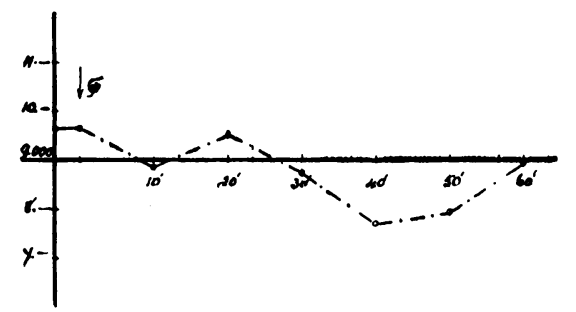

Chart 6. 
The same day $4 \cdot 5 \mathrm{~g}$. of $\mathrm{NH}_{4} \mathrm{Cl}$ was given in three doses. The following day, a dose of $3 \mathrm{~g}$. was administered an hour before the experiment.

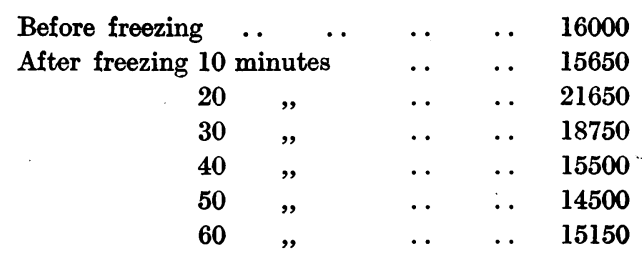

shewing rise of $29.06 \%$. (Chart 7 .)

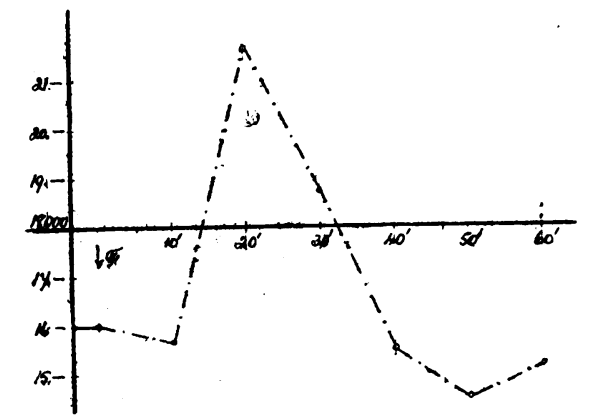

Chart 7 .

Conclusion.

From the foregoing results and examination of the literature it is clear that the reaction and the fluctuation in the number of leucocytes following irritation of the skin by freezing are a manifestation of the state of the vegetatative nervous system, and finally of the preponderance of one or the other of its parts, taking into consideration the state of the blood and viscera. Therefore I propose this procedure as a method for examining a certain part of the vegetative nervous system.

\section{REFERENCES.}

1. Müller, E. F., Munich. Med. Woch, München, 1921 and following years ; Klin. Wochensch, Berlin, 1926, V, 53, 137.

2. Filiuski, W., Compt. Rend. d.l. Soc. a. Biolog., Paris, 1924, XCI.

3. Dresel, K., Klin. Wochen, Berlin, 1924, V, 311.

4. Schill, E., Klin. Wochen, Berlin, 1927, VI, 193. 